\title{
Determinants of Maternal Renin-Angiotensin- Aldosterone-System Activation in Early Pregnancy: Insights From 2 Cohorts
}

\section{Rosalieke E. Wiegel, ${ }^{1}$ A.H. Jan Danser, ${ }^{2}$ Régine P.M. Steegers-Theunissen, ${ }^{1}$ Joop S.E. Laven, ${ }^{1}$ Sten P. Willemsen, ${ }^{1,3}$ Valerie L. Baker, ${ }^{4}$ Eric A.P. Steegers, ${ }^{1}$ and Frauke von Versen-Höynck ${ }^{5}$}

'Department of Obstetrics and Gynecology, Erasmus MC University Medical Center, 3000 CA Rotterdam, the Netherlands; ${ }^{2}$ Department of Internal Medicine, Erasmus MC University Medical Center, 3000 CA Rotterdam, the Netherlands; ${ }^{3}$ Department of Biostatistics, Erasmus MC University Medical Center, 3000 CA Rotterdam, the Netherlands; ${ }^{4}$ Department of Gynecology and Obstetrics, Johns Hopkins University School of Medicine, Lutherville, Maryland 21093, USA; and ${ }^{5}$ Department of Obstetrics and Gynecology, Hannover Medical School, Hannover 30625, Germany

\begin{abstract}
Abbreviations: $\mathrm{AMH}$, antimüllerian hormone; $\mathrm{BMI}$, body mass index; $\mathrm{CL}$, corpus luteum; COS, controlled ovarian stimulation; ET, embryo transfer; FET, frozen embryo transfer; FSH, follicle-stimulating hormone; GA, gestational age; GnRH, gonadotropin-releasing hormone; hCG, human chorionic gonadotropin; hMG, human menopausal gonadotropin; IVF, in vitro fertilization; LH, luteinizing hormone; LMP, last menstrual period; PCOS, polycystic ovary syndrome; RAAS, reninangiotensin-aldosterone-system; RAS, renin-angiotensin-system; rh, recombinant human.
\end{abstract}

Received: 19 August 2020; Accepted: 26 August 2020; First Published Online: 27 August 2020; Corrected and Typeset: 16 September 2020.

\begin{abstract}
Context: The corpus luteum (CL) secretes prorenin, renin's inactive precursor. It may thus contribute to the renin-angiotensin-aldosterone-system (RAAS) activation that is required for maternal adaptation in pregnancy. Whether this activation is disturbed in pregnancies lacking a $C L$ is unknown.

Objective: The objective of this work is to investigate maternal RAAS determinants in early pregnancy. Design and Setting: Two observational prospective cohort studies took place at 2 tertiary referral hospitals.

Patients and Intervention(s): Pregnancies $(n=277)$ were stratified by $C L$ number and in vitro fertilization (IVF) protocol: $0 \mathrm{CL}$ (programmed cycle frozen embryo transfer [FET], $\mathrm{n}=28$ ), $1 \mathrm{CL}$ (natural cycle FET, $\mathrm{n}=41$ and spontaneous conceptions, $\mathrm{n}=139$ ), and more than $1 \mathrm{CL}$ (ovarian stimulation and fresh embryo transfer, $\mathrm{n}=69$ ).

Methods: Quantification was performed for maternal prorenin, renin, and aldosterone blood levels at 5, 9, and 11 weeks of gestation.

Results: Prorenin and renin were lower in the absence of a $\mathrm{CL}$ at all time points when compared to $1 \mathrm{CL}$, whereas prorenin, renin, and aldosterone were higher in the presence of more than $1 \mathrm{CL}$ vs $1 \mathrm{CL}(P<.05)$. Ovarian stimulation with menopausal gonadotropin resulted in higher prorenin, renin, and aldosterone concentrations during the late first trimester than recombinant follicle-stimulating hormone $(P<.05)$. Prorenin, and to a lesser degree renin, correlated positively with serum progesterone and relaxin, but not serum estradiol. Total follicle diameter, body mass index (BMI), polycystic ovary syndrome (PCOS), and
\end{abstract}


antimüllerian hormone $(\mathrm{AMH})$ were additional determinants of circulating prorenin. Finally, pregnancies conceived in the absence of a $C L$ were more disposed to develop preeclampsia.

Conclusions: CL number, IVF protocol, BMI, PCOS, and AMH affect maternal RAAS activation in early pregnancy, and may thus contribute to pregnancy complications.

Freeform/Key Words: renin-angiotensin-aldosterone-system, in vitro fertilization, corpus luteum, pregnancy, prorenin, preeclampsia

An optimal ovarian endocrine function is of vital importance for normal reproductive health and the establishment of pregnancy. Essential in early pregnancy is proper corpus luteum $(\mathrm{CL})$ function, a unique temporary endocrine structure in the ovary. The CL is a major source of reproductive hormones in the periconceptional period and in early pregnancy before the endocrine function of the placenta becomes sufficiently developed to supersede it (1).

In addition to sex steroids such as estrogens, androgens, and progestagens, which are indispensable for endometrial development, implantation, placentation, and pregnancy maintenance, the CL produces important vasoactive factors like relaxin and prorenin $(2,3)$. Relaxin is a vasodilator that simultaneously contributes to cardiovascular and renal adaptation in pregnancy $(4,5)$. Prorenin, the precursor of renin, is not only released by the CL, but also during maturation of the ovarian follicle (6). Given its local synthesis, it is generally believed that there is an independent ovarian renin-angiotensin-system (RAS), resulting in high local angiotensin II concentrations $(7,8)$. Strikingly, ovarian prorenin is also released into the circulation, and may thus be part of the well-known RAS activation in the maternal circulation during early gestation $(3,9)$. Such RAS activation likely contributes to the maternal cardiovascular and hemodynamic adaptations of pregnancy because angiotensin II is an important blood pressure regulator with major effects on fluid and electrolyte balance, in part through aldosterone ("RAAS," renin-angiotensinaldosterone-system) (10).

Conrad and Baker have proposed that the CL is an important regulator of the maternal physiologic adaptation to pregnancy (1). Indeed, remarkable differences in cardiovascular, renal, and plasma volume regulation, particularly during early pregnancy, were reported between pregnancies conceived spontaneously and after in vitro fertilization (IVF) treatment, but also between the 2 main types of IVF treatment involving either controlled ovarian stimulation (COS) in the presence of multiple CL or hypothalamicpituitary suppression in absence of CL (11-14). IVF pregnancies are more frequently complicated by early and late vascular-related pregnancy complications compared to spontaneously conceived pregnancies $(15,16)$. Lately, in prospective and registry-based studies an increased risk of preeclampsia has been observed after programmed frozen embryo transfer (FET) that involved ovarian suppression and absence of a CL $(17,18)$. Taken together, these data support the importance of CL-derived endocrine factors, including those contributing to the ovarian RAS, in maternal vascular health during early pregnancy.

In light of frequent utilization of IVF treatment, more knowledge is needed about the impact of such treatment on ovarian endocrine function associated with the early adaptation to pregnancy to provide the safest treatment to patients and to improve maternal and perinatal outcome following IVF. From this background, we hypothesize that IVF treatment and fertility-specific characteristics like polycystic ovary syndrome (PCOS), body mass index (BMI), and antimüllerian hormone (AMH) affect periconception ovarian biology at least in part by modulating RAAS activation. Accordingly, the first aim of this study was to investigate maternal RAAS determinants in early pregnancy. To address this question, we used 2 large periconception cohorts, the Stanford Pregnancy Outcomes Following Infertility (POFI) Study and the Rotterdam Periconceptional Cohort (Predict) Study $(19,20)$. Importantly, von VersenHöynck et al have shown in the POFI Study that an abnormal CL number associates with increased preeclampsia risk (14). Since such pregnancy outcome data have not been linked to CL number in the Predict Study, our second aim was to confirm this relationship in the Predict cohort.

\section{Materials and Methods}

\section{Stanford Pregnancy Outcomes Following Infertility Study}

Participants were part of the Stanford Pregnancy Outcomes Following Infertility (POFI) Study (19). After approval of the study by the institutional review board at Stanford University, 878 infertile patients undergoing treatment at the Stanford Division of Reproductive Endocrinology and Infertility with sonographically confirmed viable pregnancy were recruited at 8 weeks' gestation between October 2011 and March 2014. A subcohort of 80 randomly chosen participants was selected for the analysis performed in this study. Informed consent was given for use of clinical data and previously stored serum 
samples for research purposes. One serum sample of each pregnancy was collected at 4 to 5 weeks of gestational age (GA) initially for the clinical measurement of quantitative human chorionic gonadotropin (hCG), and remnant serum not required for clinical testing was stored at $-80^{\circ} \mathrm{C}$ for later analysis. Quantification of relaxin, progesterone, and estradiol in these samples has been reported before (21). At the time of study entry, participants provided information about age, parity, ethnicity, and other demographic and clinical data using a standardized questionnaire. Maternal BMI, blood pressure, and $\mathrm{AMH}$ were determined when patients were admitted to the fertility clinic. Prenatal care and delivery records were collected from the obstetricians and hospitals that provided care for each patient.

Assisted reproduction protocols. Standard protocols were used for COS and fresh embryo transfer (ET) and FET. IVF patients received a protocol of leuprolide acetate (gonadotropin-releasing hormone [GnRH]-antagonist), or microdose leuprolide acetate and administered gonadotropins for COS. Embryo cryopreservation was performed almost always (> 95\%) at the blastocyst stage. All FET cycles were monitored by ultrasound until the endometrium reached $7 \mathrm{~mm}$ or larger. For modified natural-cycle FETs, when the lead follicle reached $18 \mathrm{~mm}$, hCG was administered in the evening, and ET was typically performed 7 days later. If a luteinizing hormone ( $\mathrm{LH}$ ) surge was detected by serum LH or an ovulation predictor kit, the transfer was performed 6 days after detection of the LH surge. Vaginal progesterone supplementation was used routinely. Programmed FET cycles used estradiol (administered orally or transdermally) and vaginal and/ or intramuscular progesterone. Absence of a developing follicle was confirmed by ultrasound for all programmed FET cycles. Spontaneous pregnancies were among women with known subfertility. GA in spontaneously conceived pregnancies was calculated based on the first day of the last menstrual period (LMP) or if known the ovulation date in spontaneously conceived pregnancies. After fresh ET GA was calculated using the ovum pick-up day plus 14 days and in pregnancies after FET, GA was defined as the transfer date plus 17 days for cleavage-stage embryos or plus 19 days for blastocyst transfers.

\section{Rotterdam Periconceptional Cohort Study (Predict Study)}

Participants were part of the Rotterdam Periconception cohort (Predict Study), an ongoing prospective, periconception cohort at the Department of Obstetrics and Gynecology of the Erasmus MC, University Medical Center, in Rotterdam, the Netherlands (20). This cohort enrolled a subcohort of 241 women between January 2017 and March 2018 with a focus on the (patho)physiology of early placental development. The Predict Study was conducted in accordance with the ethical principles for medical research set out in the Declaration of Helsinki and was approved by the medical ethical assessment committee of the Erasmus MC. Inclusion criteria are a minimum maternal age of 18 years or older, with an ongoing intrauterine singleton pregnancy of less than $10^{0}$ weeks of gestation. Exclusion criteria for the present study are pregnancies ending in miscarriages, oocyte donation, withdrawal, and fetuses or neonates with congenital malformations. The study involves questionnaires at study entry and throughout gestation, serial 3-dimensional ultrasound measurements, and serial blood withdrawal (EDTA tubes) at 9 and 11 weeks' GA. Maternal blood pressure, weight, and height were measured at the study entry visit. Infertility diagnosis, treatment, and clinical measurement of quantitative AMH in serum was obtained from medical records. Written informed consent was obtained from all study participants and partner at enrollment.

Assisted-reproduction protocols. Standard protocols were used for COS, ovum pick-up, IVF treatment, and assessment of embryo morphology $(22,23)$. IVF patients received either GnRH agonists (Decapeptyl or Lucrin) or antagonists (Orgalutran, Cetrotide, or Fyremadel). Women received COS with either Menopur, a highly purified, urine-derived, human menopausal gonadotropin (hMG, containing follicle-stimulating hormone $[\mathrm{FSH}], \mathrm{LH}$, and hCG) or recombinant human (rh)FSH (Bemfola, Gonal-F, or Rekovelle). For modified natural-cycle FETs, a frozenthawed ET was performed 4 days after spontaneous ovulation, detected by urinary LH testing. In programmed FET cycles the endometrium was prepared using estrogens followed by increasing dosages of intravaginal progesterone and daily injections of a GnRH agonist. All FET cycles were monitored by ultrasound until the endometrium reached $4 \mathrm{~mm}$ or larger. Transfer of the frozen-thawed embryo occurred after 15 days of endometrial preparation. Spontaneous pregnancies in this cohort occurred in women with and without known subfertility. GA was calculated based on the first day of LMP in spontaneously conceived pregnancies or the ovum pick-up day plus 14 days for pregnancies after fresh ET. In pregnancies after FET, GA was defined as the transfer date plus 19 days. In case of an irregular menstrual cycle, unknown LMP, or the GA based on LMP deviated more than 6 days from GA based on crown-rump-length, the GA was based on crown-rump-length. 


\section{Classification of the 2 study groups}

Participants were grouped according to CL number at conception: (1) $0 \mathrm{CL}$, from FET in a programmed cycle, (2) 1 $\mathrm{CL}$, spontaneous conceptions, including intrauterine insemination (IUI) and hormonal treatment or FET in a modified natural ovulatory cycle, (3) more than $1 \mathrm{CL}$, ovarian stimulation with fresh ET. The CL status at conception in pregnancies conceived by fresh ET is based on number of retrieved eggs during ovum pick-up (median: 11.0 [interquartile range, 7-16]).

\section{Renin, prorenin, and aldosterone measurements}

All measurements were performed at the Department of Internal Medicine of the Erasmus MC, making use of either serum (POFI Study) or EDTA plasma (Predict Study). Renin and prorenin concentrations were measured by an immunoradiometric assay (Cisbio) using an active sitedirected radiolabeled antibody that recognizes renin only. Prorenin concentrations are calculated by subtracting renin from total renin measured after activating prorenin with aliskiren (24). Aldosterone concentrations were measured by solid-phase radioimmunoassay (Demeditec Diagnostics).

\section{Hypertensive pregnancy outcomes in the Predict Study}

To verify whether the previously observed increased rate of preeclampsia in pregnancies conceived in the absence of a CL (14) also applies to the Predict Study, we analyzed clinical hypertensive pregnancy outcomes according to number of CL in participants included between January 2012 and December 2016 in this cohort. Postpartum filled-out questionnaires were checked with medical records. Hypertensive disorders were defined according to the current American Congress of Obstetricians and Gynecologists guidelines (25).

\section{Statistical analysis}

Data are presented as mean \pm SD for normal distributed variables and median and interquartile range for skewed variables. Analysis was performed by analysis of variance and Kruskal-Wallis test, respectively. Categorical characteristics, described using frequencies (proportions), were compared by chi-square test. Log-transformed RAAS component concentrations in the various groups were compared by MannWhitney $U$ test. Based on a previous study by Verdonk and colleagues (26) on RAAS component levels in pregnant women, a minimum number of 8 per group was calculated to be sufficient to detect a $50 \%$ difference in renin, prorenin, and aldosterone with $80 \%$ power and $5 \%$ significance. Spearman $\rho$ rank correlation coefficient $(R)$ was used to evaluate correlations between hormonal levels. The relationship between fertility-specific characteristics-BMI, PCOS, $\mathrm{AMH}$, number of follicles, retrieved oocytes on the punction date after ovum pick-up, and total follicle diameter (determined the day of ovulation triggering, and representing the sum of the diameters of all follicles), and prorenin concentration- was studied by multivariable linear regression analysis after adjusting for relevant confounders (CL number, maternal age, BMI, stimulation treatment, and/or treatment dose). Hypertensive pregnancy outcomes were analyzed by CL number and compared by the chi-square test. Multivariable logistic regression was used with preeclampsia as outcome variable and CL number as predictor. The model was adjusted for maternal age, BMI, parity, smoking, and chronic hypertension. All analyses were performed using $\mathrm{R}$ ( $\mathrm{R}$ for Windows, version 3.5; R Core Team). A $P$ value of less than .05 was considered significant.

\section{Results}

\section{Baseline characteristics}

For this study 80 participants from the POFI Study were included and 241 pregnancies from the Predict Study were enrolled. After excluding 27 pregnancies ending in miscarriage, 8 with congenital malformation, 4 from oocyte donation, and 5 for which blood was not available in the first trimester, 75 (POFI) and 202 (Predict) pregnancies were eligible for analysis. Table 1 shows for both study groups the maternal baseline characteristics stratified for CL number at the moment of conception. All CL groups were comparable with regard to age, preconception blood pressure, GA at the moment of blood draw, ethnicity, and smoking. In the Predict Study preconception alcohol use was higher in the $0 \mathrm{CL}$ group and the $1 \mathrm{CL}$ group displayed a higher BMI. There was a higher rate of nulliparous pregnancies in the $0 \mathrm{CL}$ and more than $1 \mathrm{CL}$ groups compared to the 1 CL group. The Predict Study contained more women with PCOS, particularly in the 0 CL group.

\section{First-trimester maternal renin-angiotensin- aldosterone-system concentrations}

Maternal concentrations of renin, prorenin, and aldosterone by CL number at conception are presented in Fig. 1. Renin and prorenin were significantly lower in the $0 \mathrm{CL}$ group at all time points when compared to the 1 CL group. Renin, prorenin, and aldosterone at 9 and 11 weeks were significantly higher in the presence of more than $1 \mathrm{CL}$ when 
Table 1. Baseline demographic and clinical characteristics of Pregnancy Outcomes Following Infertility and Predict Study participants by corpus luteum number

\begin{tabular}{|c|c|c|c|c|}
\hline POFI Study group & $0 \mathrm{CL}(\mathrm{n}=20)$ & $1 \mathrm{CL}(\mathrm{n}=37)$ & $>1 \mathrm{CL}(\mathrm{n}=18)$ & $P$ \\
\hline \multicolumn{5}{|l|}{ Maternal characteristics } \\
\hline Maternal age, y & $34.2 \pm 3.8$ & $34.1 \pm 4.3$ & $34.3 \pm 4.5$ & .98 \\
\hline GA blood draw, 5 wks, d & $28.0(28.0-29.0)$ & $29.0(27.0-31.0)$ & $27.0(27.0-28.8)$ & .08 \\
\hline Nulliparous (n, \%) & $17(85.0)$ & $17(45.6)$ & $15(83.3)$ & $<.01$ \\
\hline Ethnicity, (n, \% Non-Hispanic or Non-Latino) & $18(90)$ & $36(97.3)$ & $18(100)$ & .25 \\
\hline $\mathrm{BMI}$ at study entry, $\mathrm{kg} / \mathrm{m}^{2}$ & $23.6(20.7-26.3)$ & $23.7(21.0-26.7)$ & $22.4(19.9-24.9)$ & .50 \\
\hline MAP at study entry & $83.2(6.2)$ & $86.0(10.6)$ & $84.4(10.0)$ & .55 \\
\hline $\operatorname{PCOS}(\mathrm{n}, \%)$ & $5(25.0)$ & $0(0.0)$ & $2(11.1)$ & .07 \\
\hline Lifestyle & & & & .70 \\
\hline Preconceptional alcohol use (n, \%) & $1(8.3)$ & $0(0.0)$ & $1(10.0)$ & .36 \\
\hline Preconceptional smoking (n, \%) & $1(5.0)$ & $3(8.1)$ & $2(11.1)$ & .79 \\
\hline \multicolumn{5}{|l|}{ Biochemical measurements } \\
\hline Relaxin, pg/mL & $7.8(7.8-7.8)^{a}$ & $221(126-280)$ & $294(161-448)$ & $<.001$ \\
\hline Progesterone, $\mathrm{ng} / \mathrm{mL}$ & $5.6(4.1-8.2)$ & $18(14-24)$ & $57(39-103)$ & $<.001$ \\
\hline Estradiol, pg/mL & $194(146-375)$ & $162(116-347)$ & $470(210-755)$ & .05 \\
\hline Preconceptional AMH & $3.3(1.3-7.2)$ & $3.0(3.0-3.0)$ & $1.8(1.0-3.5)$ & .70 \\
\hline Predict Study group & $0 \mathrm{CL}(\mathrm{n}=8)$ & $1 \mathrm{CL}(\mathrm{n}=143)$ & $>1 \mathrm{CL}(\mathrm{n}=51)$ & $P$ \\
\hline \multicolumn{5}{|l|}{ Maternal characteristics } \\
\hline Maternal age, y & $30.5 \pm 3.9$ & $32.0 \pm 4.4$ & $33.0 \pm 4.7$ & 0.25 \\
\hline GA blood draw, 9 wks, d & $67.5(65.5-68.3)$ & $65.0(64.0-67.5)$ & $66.0(65.0-67.0)$ & .14 \\
\hline GA blood draw, 11 wks, d & $81.5(79.5-82.3)$ & $79.0(78.0-82.0)$ & $79.0(78.0-82.0)$ & .39 \\
\hline Nulliparous (n, \%) & $6(75.0)$ & $68(47.6)$ & $42(82.4)$ & $<.01$ \\
\hline Ethnicity $(\mathrm{n}, \%)$ & & & & .24 \\
\hline Dutch & $6(75.0)$ & $104(75.4)$ & $46(90.2)$ & \\
\hline Western & $0(0.0)$ & $5(3.6)$ & $1(2.0)$ & \\
\hline Non-Western & $2(25.0)$ & $29(21.0)$ & $4(7.8)$ & \\
\hline $\mathrm{BMI}$ at study entry, $\mathrm{kg} / \mathrm{m}^{2}$ & $21.4(20.9-22.8)$ & $25.2(22.6-29.2)$ & $23.9(21.8-27.4)$ & .02 \\
\hline MAP at study entry & $80.8 \pm 12.1$ & $80.7 \pm 6.8$ & $79.6 \pm 7.1$ & .74 \\
\hline $\operatorname{PCOS}(n, \%)$ & $5(62.5)$ & $21(14.7)$ & $15(29.4)$ & $<.01$ \\
\hline \multicolumn{5}{|l|}{ Lifestyle } \\
\hline Preconceptional alcohol use (n, \%) & $5(62.5)$ & $42(29.4)$ & $10(19.6)$ & .04 \\
\hline Preconceptional smoking (n, \%) & $1(12.5)$ & $21(14.7)$ & $5(9.8)$ & .68 \\
\hline \multicolumn{5}{|l|}{ Biochemical measurements } \\
\hline Preconceptional AMH & $4.0(3.5-7.7)$ & $9.3(3.9-12.9)$ & $4.2(2.9-7.0)$ & .32 \\
\hline
\end{tabular}

Data are presented as mean \pm SD or median (interquartile range). Relaxin, progesterone, and estradiol levels were available in 47 (19, 16 , and 12$), 46$ (19, 15 and 12), and 45 (19, 15 and 11) women, respectively.

Abbreviations: AMH, antimüllerian hormone; BMI, body mass index; CL, corpus luteum; GA, gestational age; MAP, mean arterial pressure; PCOS, polycystic ovary syndrome; POFI, Pregnancy Outcomes Following Infertility.

${ }^{a}$ All relaxin levels in the 0 CL group were below the detection limit $(7.8 \mathrm{pg} / \mathrm{mL})$.

compared to $1 \mathrm{CL}$. Although the same difference was observed for renin and prorenin at 5 weeks, this was not significant. Within the 1 CL group, no differences were observed for renin, prorenin, and aldosterone between FET in a natural cycle and spontaneous conception (data not show). The renin/total renin ratio decreased by increasing CL number, although this was significant at 11 weeks only when comparing the more than $1 \mathrm{CL}$ group with the $1 \mathrm{CL}$ group $(P=.01)$. The renin/total renin ratio was higher in the 5 -week serum samples than in the 9- and 11-week plasma samples $(P<.001)$. The lowest renin/total renin ratio was observed at 9 weeks in the 7 women in the Predict Study who later developed preeclampsia (5.56 [5.39-5.73] vs 8.02 [6.47-9.76] in the 195 nonpreeclamptic cases; $P=.04)$. The aldosterone/renin ratio was lower in the $1 \mathrm{CL}$ group vs the 0 CL group $(P<.05)$, and increased again in the more than 1 CL group compared to the $1 \mathrm{CL}$ group $(P<.01)$. Aldosterone levels were higher in the more than $1 \mathrm{CL}$ group vs the $1 \mathrm{CL}$ group $(P<.001)$. Renin correlated more strongly with aldosterone in the more than $1 \mathrm{CL}$ group than in the $1 \mathrm{CL}$ group (Fig. 2). In the 0 CL group, no significant correlation was observed between renin and aldosterone.

Relaxin, progesterone, and estradiol levels are shown in Table 1 , and have been derived from a previously published 

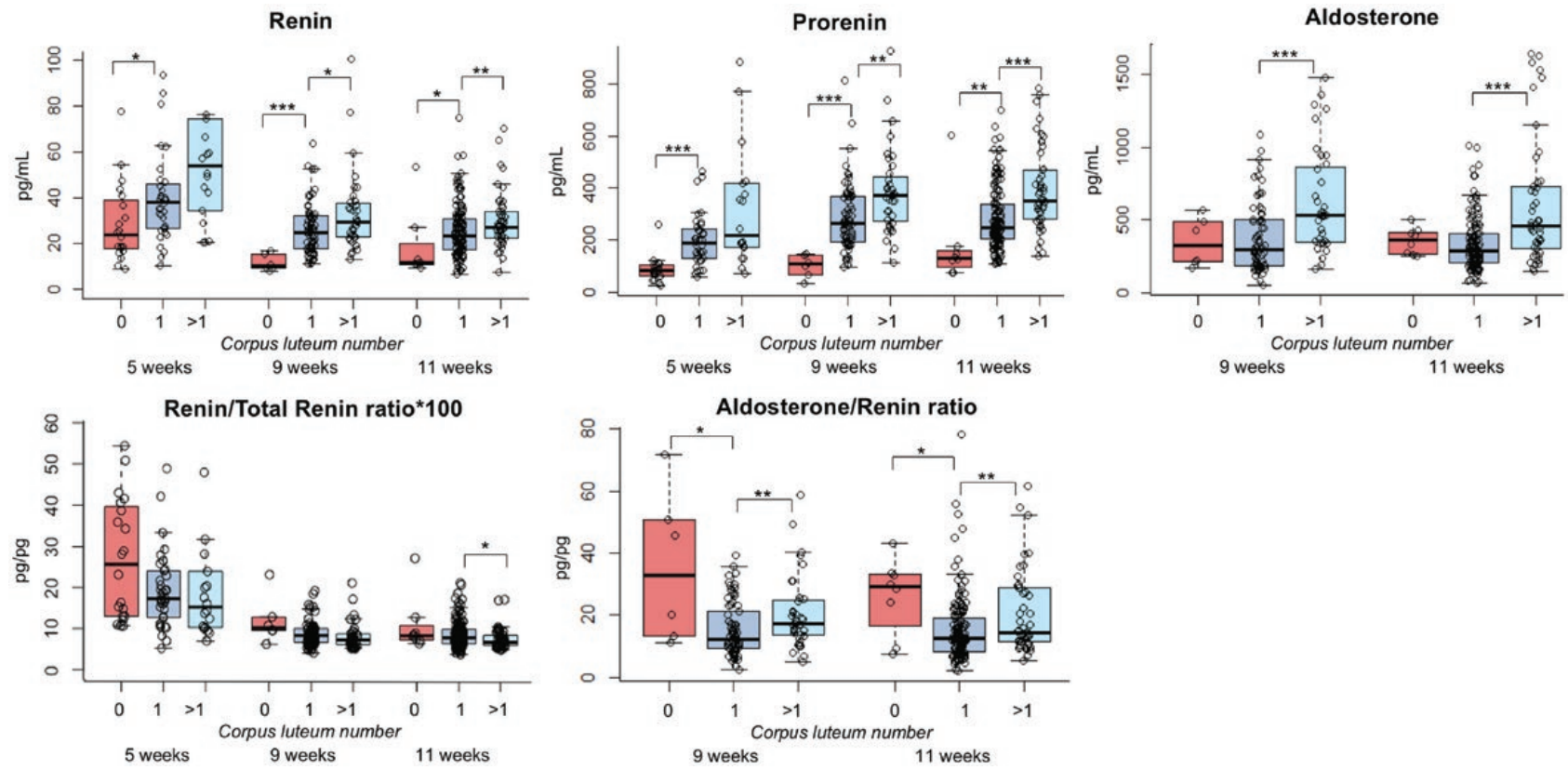

Figure 1. Comparison of maternal renin, prorenin, aldosterone, renin/total renin ratio, and aldosterone/renin ratio concentrations in first trimester of pregnancy at 5,9 , and 11 weeks of gestation in women conceived by different numbers of corpora lutea (CL) at conception (5 weeks: $0 \mathrm{CL}$ [ $\mathrm{n}=20], 1$ $\mathrm{CL}[\mathrm{n}=37]$ or $>1 \mathrm{CL}[\mathrm{n}=18], 9$ weeks: $0 \mathrm{CL}[\mathrm{n}=6], 1 \mathrm{CL}[\mathrm{n}=70]$ or $>1 \mathrm{CL}[\mathrm{n}=35]$ and 11 weeks: $0 \mathrm{CL}[\mathrm{n}=8], 1 \mathrm{CL}[\mathrm{n}=138]$ or $>1 \mathrm{CL}[\mathrm{n}=46])$. Boxplots present median, 10th, 25th, 75th, and 90th percentiles. Log-transformed renin-angiotensin-aldosterone-system component concentrations in the various groups were compared by Mann-Whitney $U$ test. ${ }^{*} P$ less than $.05,{ }^{*} P$ less than $.01,{ }^{* *} P$ less than .001 .

$0 \mathrm{CL}$

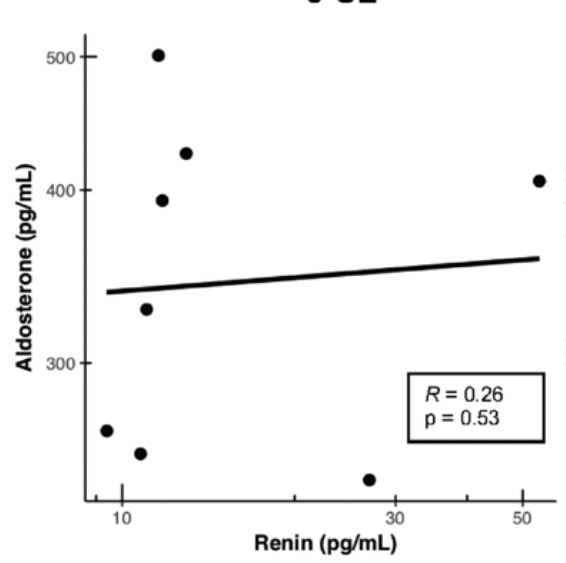

$1 \mathrm{CL}$

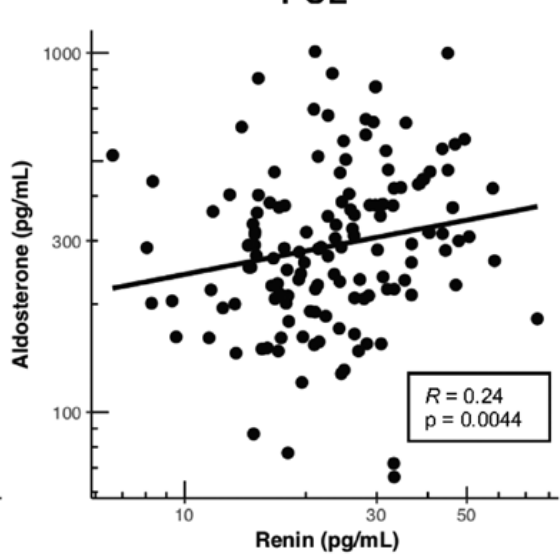

$>1 \mathrm{CL}$

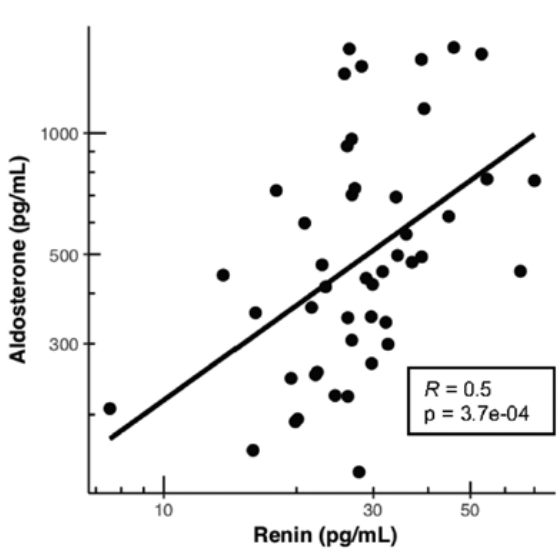

Figure 2. Correlations by Spearman rank test between maternal plasma aldosterone and plasma renin concentrations at 11 weeks of gestation in women conceived by different numbers of corpora lutea (CL) at conception (11 weeks: $0 \mathrm{CL}[\mathrm{n}=8], 1 \mathrm{CL}[\mathrm{n}=138]$, or $>1 \mathrm{CL}[\mathrm{n}=46]$ ).

report on a POFI Study subset (21). Relaxin was below the detection limit in all women without a CL. Both relaxin and progesterone, but not estradiol, correlated significantly with renin and prorenin (Fig. 3). Correlations were much stronger with prorenin than renin.

\section{Impact of in vitro fertilization protocol on renin-} angiotensin-aldosterone-system concentrations

In the Predict Study IVF patients received diverse drugs for COS. Women who conceived with fresh ET after COS with $\mathrm{hMG}(\mathrm{n}=21)$ showed significantly higher renin, prorenin, and aldosterone concentrations at 9 and 11 weeks than those stimulated with $\mathrm{rhFSH}(\mathrm{n}=26$; Table 2 ). There was no difference in RAAS component concentrations concerning the use of GnRH (ant)agonists (data not shown), nor did the characteristics of the women treated with hMG or rhFSH differ (data not shown).

\section{Prorenin determinants: periconceptional} maternal characteristics and fertility parameters

Given the previously described relationship between RAAS parameters and CL number, and because infertility 

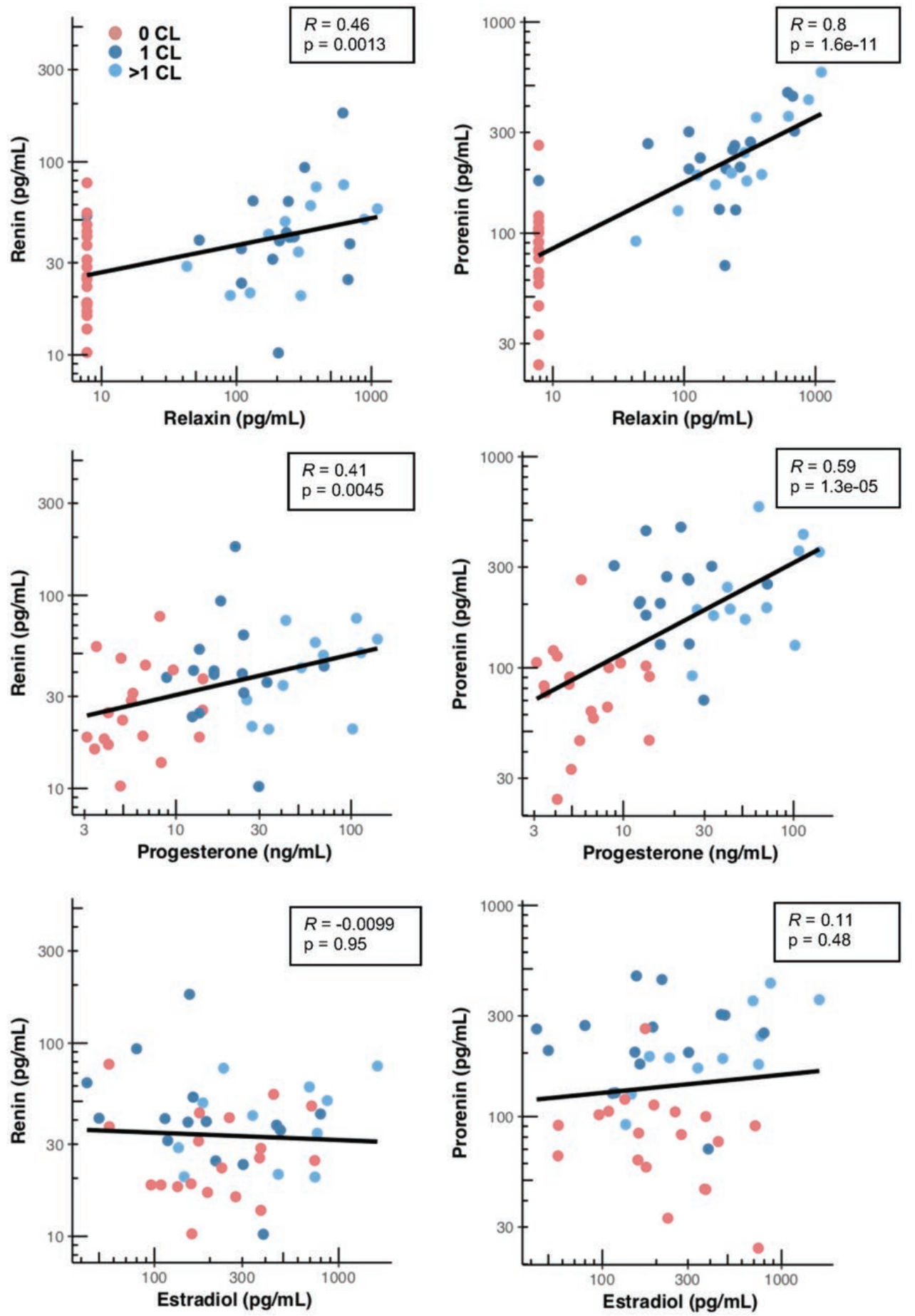

Figure 3. Correlations by Spearman rank test between relaxin $(n=47)$, progesterone $(n=46)$, estradiol $(n=45)$, and renin and prorenin concentrations in serum samples at 5 weeks of gestation in women conceived by different numbers of corpora lutea (CL) at conception.

(requiring IVF treatment) increases with age, we studied the association between prorenin and BMI in the entire study group after adjusting CL number and maternal age (27). This revealed a negative relationship between BMI and the prorenin concentrations at 9 and 11 weeks' gestation (Table 3). When studying the prorenin association with AMH and PCOS, we additionally adjusted for BMI (on top of CL number and age), given the well-known relationship between BMI, AMH, and PCOS $(28,29)$. It was then found that AMH and PCOS associated positively with the prorenin concentrations at 11 weeks (Table 3). Finally, using the group with the largest n-number (11-week cohort, $\mathrm{n}=192$ ), we incorporated all prorenin-determining parameters (CL number, BMI, AMH, and PCOS) and age 
Table 2. Maternal renin-angiotensin-aldosterone-system concentrations of pregnancies conceived by fresh embryo transfer (> 1 corpora lutea) after controlled ovarian stimulation by gonadotropin used

\begin{tabular}{lccc}
\hline & rhFSH $(\mathbf{n}=\mathbf{2 6})$ & hMG $(\mathbf{n}=\mathbf{2 1})$ & \\
\hline 9 wks' GA & & & \\
Renin, pg/mL & $24.1(22.2-31.8)$ & $35.7(29.5-44.5)$ & .01 \\
Prorenin, pg/mL & $353(244-396)$ & $398(352-533)$ & .03 \\
Aldosterone, pg/mL & $394(327-538)$ & $848(534-987)$ & .002 \\
Renin/Total renin ratio, $\times 100$ & $6.92(5.86-8.87)$ & $7.66(6.29-8.80)$ & .64 \\
Aldosterone/Renin ratio & $17.1(13.2-20.5)$ & $20.0(15.4-31.0)$ & .26 \\
11 wks' GA & & $401(333-590)$ \\
Renin, pg/mL & $26.1(20.5-28.7)$ & $621(392-1221)$ \\
Prorenin, pg/mL & $334(264-409)$ & $6.51(6.07-7.22)$ \\
Aldosterone, pg/mL & $358(235-538)$ & $17.8(14.0-36.0)$ \\
Renin/Total renin ratio, $\times 100$ & $6.52(5.61-7.65)$ & .02 \\
Aldosterone/Renin ratio & $13.3(10.8-24.5)$ & .045 \\
\hline
\end{tabular}

Median value (interquartile range) are shown; Mann-Whitney $U$ test was performed.

Abbreviations: GA, gestational age; hMG, human menopausal gonadotropin; rhFSG, recombinant human follicle-stimulating hormone.

Table 3. Determinants of circulating prorenin. Maternal characteristics refer to total study group (0 corpus luteum $[\mathrm{CL}], 1 \mathrm{CL}$, and $>1 \mathrm{CL}$ ), and fertility parameters refer to pregnancies conceived by fresh embryo transfer (> $1 \mathrm{CL}$ group)

\begin{tabular}{|c|c|c|c|c|c|c|}
\hline & \multicolumn{2}{|l|}{$\begin{array}{c}5 \text { wks' GA log } \\
\text { (prorenin), pg/mL }\end{array}$} & \multicolumn{2}{|l|}{$\begin{array}{c}9 \text { wks' GA log } \\
\text { (prorenin), pg/mL }\end{array}$} & \multicolumn{2}{|l|}{$\begin{array}{l}11 \text { wks' GA log } \\
\text { (prorenin), pg/mL }\end{array}$} \\
\hline & $\beta(95 \% \mathrm{CI})$ & $P$ & $\beta(95 \% \mathrm{CI})$ & $P$ & $\beta(95 \% \mathrm{CI})$ & $P$ \\
\hline Maternal characteristics & $\mathrm{N}=75$ & & $\mathrm{~N}=111$ & & $\mathrm{~N}=192$ & \\
\hline Log, BMI, $\mathrm{kg} / \mathrm{m}^{2 a}$ & $-.307(-1.043$ to .429$)$ & .41 & $-.915(-1.340$ to -.492$)$ & $<.001$ & $-.786(-1.096$ to -.476$)$ & $<.001$ \\
\hline $\operatorname{PCOS}^{b}$ & $.389(-.060$ to .839$)$ & .09 & $-.028(-.241$ to .186$)$ & .80 & $.157(.003$ to .310$)$ & .04 \\
\hline $\log \mathrm{AMH}^{b}$ & $.128(-.108$ to .363$)$ & .26 & $.196(-.124$ to .516$)$ & .21 & $.235(.035$ to .435$)$ & .02 \\
\hline Fertility parameters $^{c}$ & $\mathrm{~N}=18$ & & $\mathrm{~N}=35$ & & $\mathrm{~N}=46$ & \\
\hline No. of follicles & $-.206(-1.126$ to .714$)$ & .64 & $.148(-.329$ to .624$)$ & .52 & $.318(-.144$ to .780$)$ & .17 \\
\hline Total follicle diameter & $-.128(-1.085$ to .829$)$ & .78 & $.248(-.201$ to .698$)$ & .26 & $.402(-.004$ to .809$)$ & .05 \\
\hline No. of oocytes & $-.198(-.513$ to .117$)$ & .21 & $-.070(-.416$ to .194$)$ & .70 & $-.096(-.474$ to .282$)$ & .60 \\
\hline Fertility parameters in non-PCOS & $\mathrm{N}=16$ & & $\mathrm{~N}=26$ & & $\mathrm{~N}=35$ & \\
\hline No. of follicles & $-.108(-1.087$ to .871$)$ & .82 & $.387(-.310$ to 1.083$)$ & .25 & $.472(-.140,1.085)$ & .12 \\
\hline Total follicle diameter & $-.044(-1.050$ to .963$)$ & .93 & $.509(-.103$ to 1.121$)$ & .09 & $.625(.113$ to 1.136$)$ & .02 \\
\hline No. of oocytes & $-.118(-.442$ to .206$)$ & .47 & $-.211(-.669$ to .246$)$ & .33 & $-.224(-.704$ to .257$)$ & .34 \\
\hline
\end{tabular}

The table shows the effect estimates of the linear models for the association between maternal characteristics in the total study group and fertility parameters during ovum pick-up from pregnancies conceived by fresh embryo transfer (> 1 CL group) and maternal prorenin concentrations at 5, 9, and 11 weeks of gestation. Abbreviations: AMH, antimüllerian hormone; BMI, body mass index; CL, corpus luteum; GA, gestational age; PCOS, polycystic ovary syndrome.

${ }^{a}$ Model adjusted for CL number at conception and maternal age.

${ }^{b}$ Model adjusted for CL number at conception, maternal age, and BMI.

${ }^{c}$ Model adjusted for stimulation method, dose, and maternal age. Fertility parameters were log-transformed.

into a multiple linear regression model. Under those conditions, only AMH, maternal age, and CL number remained independent determinants of $\log$ [prorenin] $(\log [\mathrm{AMH}] \beta$ : $.210, P=.035$; age $\beta: .033, P=.014 ; 1$ CL group $\beta: .483$, $P=.033 ;>1$ CL group $\beta: 1.070, P<.001)$.

In the more than $1 \mathrm{CL}$ group, after adjusting for stimulation method, dose, and maternal age, total follicle diameter prior to ovum pick-up associated positively with prorenin concentrations at 11 weeks, albeit not significantly (see Table 3 ). This association became significant when excluding women with PCOS. The latter outcome was unaltered after additional adjusting for BMI and AMH (total follicle diameter $\beta$ : .542 [95\% CI: 0.036$1.048], P=.037)$. No linear relation was found between the number of follicles or retrieved eggs and maternal prorenin concentrations during the first trimester.

\section{Hypertensive pregnancy outcomes in the predict study}

Of the 868 singleton live birth pregnancies that were included between January 2012 and December 2016 in 
the Predict Study, 29 pregnancies conceived in the absence of a CL, 672 with $1 \mathrm{CL}$ and 167 with multiple CL. Women who conceived through COS with fresh ET ( $>1$ CL) were more often nulliparous (Table 4). Women who conceived with $1 \mathrm{CL}$ displayed a higher preconception BMI $(24.6 \pm 8.5)$ and smoking status and more often had a history of hypertensive disease in previous pregnancy. Women who conceived in the absence of a CL were slightly older and more often hypertensive before pregnancy.

The incidence of developing gestational hypertension, preeclampsia, HELLP (hemolysis, elevated liver enzymes, and low platelets) syndrome, and eclampsia was higher after conception in the absence of a CL compared with 1 $\mathrm{CL}$ and more than $1 \mathrm{CL}$ (Table 5). The multivariate adjusted odds ratio for preeclampsia risk in the absence of a CL compared with 1 CL was 4.23 (95\% CI, 1.17-12.94; $P=.02)$.

\section{Discussion}

In this combined study of 2 periconception cohorts we demonstrate that, during the first trimester, pregnancies conceived in the absence of a CL showed lower circulating renin and prorenin concentrations compared to 1 $\mathrm{CL}$, whereas renin, prorenin, and aldosterone concentrations were higher in the presence of more than $1 \mathrm{CL}$ vs 1 CL. Additional maternal RAAS determinants in early pregnancy were type of ovarian stimulation, BMI, PCOS, $\mathrm{AMH}$, and total follicle diameter. Secondly, an abnormal CL number associates with increased preeclampsia risk.

RAAS data were consistent at 3 different time points, and agree with the concept that prorenin is produced by the $\mathrm{CL}$, resulting in higher prorenin levels at higher CL numbers (30-32). This also seemed to be the case for renin. However, some caveats need to be considered. First, serum samples were available for the POFI Study (5 weeks) and plasma samples for the Predict Study (9 and 11 weeks), while all samples had been stored for more than 12 months at $-80^{\circ} \mathrm{C}$. Depending on the sampling conditions, $\mathrm{pH}$, and temperature, prorenin's conformation may alter from closed (with the prosegment covering the active site) to open (with the active site being accessible to antibodies applied in the renin assay) (33). The consequence of this "nonproteolytic" prorenin activation is that some prorenin may be recognized as renin in the renin immunoradiometricassay, thereby artificially elevating renin and lowering prorenin. It is important to note that prorenin levels are at least one order of magnitude higher than renin levels. Hence, such activation has major consequences for renin, but hardly affects prorenin.

To address this issue, we calculated the renin/total renin ratio. This ratio differed between serum and plasma samples, confirming that sampling differences are likely to affect prorenin. The ratio decreased at increasing CL levels at all time points. This is suggestive for a relatively higher rise in prorenin than renin, but still does not exclude that some prorenin is recognized as renin. Therefore, additionally, at 9 and 11 weeks, we determined the aldosterone/renin ratio. This ratio decreased between $0 \mathrm{CL}$ and $1 \mathrm{CL}$, implying either that aldosterone became dependent on factors other than renin, or that indeed renin levels were higher. The aldosterone/renin ratio increased again in the more than 1 CL group compared to the 1 CL group. Simultaneously, aldosterone levels nearly doubled in women with more than $1 \mathrm{CL}$. Because the renin-aldosterone correlation was strongest in the latter group, suggestive for renin (via angiotensin II) being the main determinant of this aldosterone

Table 4. Baseline demographic and clinical characteristics of study participants included January 2012 to December 2016 in the Predict Study $(\mathrm{n}=868)$

\begin{tabular}{|c|c|c|c|c|}
\hline Predict Study participants, 2012 to 2016 & $0 \mathrm{CL}(\mathrm{n}=29)$ & $1 \mathrm{CL}(\mathrm{n}=672)$ & $>1 \mathrm{CL}(\mathrm{n}=167)$ & $P$ \\
\hline Age, y & $34.9 \pm 5.1$ & $31.8 \pm 4.7$ & $33.4 \pm 4.2$ & $<.001$ \\
\hline Age $>40 y,(n, \%)$ & $2(8.3)$ & $19(2.9)$ & $9(5.7)$ & .10 \\
\hline BMI, $\mathrm{kg} / \mathrm{m}^{2}$ & $22.8 \pm 10.3$ & $24.6 \pm 8.5$ & $22.8 \pm 9.0$ & .04 \\
\hline $\mathrm{BMI}>30,(\mathrm{n}, \%)$ & $6(20.7)$ & $127(18.9)$ & 19 (11.4) & .16 \\
\hline Nulliparous, (n, \%) & $18(62.1)$ & $254(37.8)$ & $115(68.9)$ & $<.001$ \\
\hline Ethnicity, (n, \%) & & & & .28 \\
\hline Dutch & $27(93.1)$ & $479(79.8)$ & $127(78.4)$ & \\
\hline Western & $1(3.4)$ & $29(4.8)$ & $14(8.6)$ & \\
\hline Non-Western & $1(3.4)$ & $91(15.2)$ & $21(13.0)$ & \\
\hline Maternal smoking in pregnancy, $(n, \%)$ & $0(0.0)$ & $110(16.4)$ & $21(12.6)$ & $<.001$ \\
\hline History of chronic hypertension, $(\mathrm{n}, \%)$ & $2(6.9)$ & $28(4.2)$ & $1(0.6)$ & .04 \\
\hline History of hypertensive disease in previous pregnancy, $(\mathrm{n}, \%)$ & $0(0.0)$ & $49(11.4)$ & $3(4.6)$ & .09 \\
\hline
\end{tabular}

Data are presented as mean \pm SD or as number (percentage of total).

Abbreviations: BMI, body mass index; CL, corpus luteum. 
Table 5. Hypertensive disorders of pregnancy stratified by corpus luteum category for participants in the Predict Study, January 2012 to December $2016(n=868)$

\begin{tabular}{|c|c|c|c|c|}
\hline Hypertensive disorder of pregnancy & $0 \mathrm{CL}(\mathrm{n}=29)$ & $1 \mathrm{CL}(\mathrm{n}=672)$ & $>1 \mathrm{CL}(\mathrm{n}=167)$ & $P$ \\
\hline Gestational hypertension & $6(20.7)$ & $50(7.4)$ & $6(3.6)$ & .007 \\
\hline Preeclampsia & $5(17.2)$ & $33(4.9)$ & $5(3.0)$ & .008 \\
\hline Early-onset preeclampsia (<34 wks’ GA) & $1(20.0)$ & $12(36.4)$ & $1(20.0)$ & .89 \\
\hline HELLP syndrome & $1(3.4)$ & $2(0.3)$ & 0 & .02 \\
\hline Chronic hypertension with superimposed preeclampsia & 0 & $11(1.7)$ & $1(0.6)$ & .50 \\
\hline Eclampsia & $1(3.4)$ & $2(0.3)$ & 0 & .02 \\
\hline
\end{tabular}

Data are presented for singleton live births in number of pregnancies (percentage of total). Chi-square test was performed. Abbreviations: CL, corpus luteum; GA, gestational age; HELLP, hemolysis, elevated liver enzymes, and low platelets.

increase, it is reasonable to assume that the aldosterone increase is indeed largely due to RAS activation. Therefore, the renin increase in relation to $\mathrm{CL}$ number is likely to be real, and not the simple consequence of in vitro prorenin activation. In fact, renin increases in pregnancy are well established (34), although information on such increases in early pregnancy is scarce. Renin increases are more modest in women with preeclampsia, a condition that is known to occur more often in the 0 CL group $(14,35)$. Given that the CL releases largely, if not exclusively, prorenin (31), any change in renin must originate in the kidney. To what degree the ovarian prorenin release and the renal renin regulation are related is currently unknown.

Prorenin release from the CL is known to be driven by hCG $(30,32)$. This likely explains the larger increase in prorenin with hMG (which contains hCG, in addition to FSH and LH) compared with rhFSH. Yet, hMG also affected renin and aldosterone much more strongly than rhFSH. Why these effects could still be seen at 9 and 11 weeks after stimulation cannot be concluded from this study. Possibly, the changes induced by hCG, FSH, and/or LH on RAAS component expression involve long-lasting induced epigenetic effects, with perhaps a dose-dependent effect of hCG on DNA methyl transferase protein expression after $\operatorname{COS}(36,37)$.

Prorenin is synthesized by theca cells (38). The theca endocrine and vascular cells develop into the granulosa cell layer, which underlies the formation of the CL (39). This might explain why we observed a positive association between prorenin levels and total follicle diameter at 11 weeks. Importantly, this association was not significant in women with PCOS, most likely because their follicles are known to function aberrantly (40). In addition to prorenin, the CL also synthesizes estradiol and progesterone, and it is the sole secretor of relaxin in early pregnancy (11). From this perspective it is not surprising that prorenin levels correlated positively with the levels both of relaxin and progesterone. In fact, relaxin has been reported to be a direct stimulator of prorenin synthesis and release (41). Moreover, as a potent vasodilator, capable of increasing glomerular filtration (5), relaxin would also be expected to activate the circulating RAS, thus explaining its relationship with the levels of renin. Similarly, progesterone, an inhibitor of the sodium-retaining effects of aldosterone, is thought to increase renin secretion (42). In contrast, estradiol is a well-known stimulator of angiotensinogen (43). Consequently, given the inverse relationship between angiotensinogen and renin, no positive correlation between estradiol and renin would be expected. Yet, when simultaneously considering the increase in angiotensinogen by comparing plasma renin activity (which reflects renin plus angiotensinogen) and estradiol in pregnant women, a positive relationship can be observed (42).

The exact role of prorenin in pregnancy and in the ovary in particular remains elusive. Although binding to the (pro) renin receptor initially has been suggested to allow prorenin to display activity, more recent studies demonstrated that such interaction required prorenin concentrations that are unlikely to ever occur in humans (44). Hence to what degree prorenin truly determines ovarian angiotensin generation is still unknown.

Women with PCOS display increased prorenin levels (45). This might be due to enhanced thecal activity, either as a result of the elevated LH levels in this condition (46) or an increased sensitivity of thecal cells to gonadotropins $(47,48)$. We were able to confirm PCOS as a determinant of plasma prorenin levels, and this was also true for $\mathrm{AMH}$, an important biomarker in PCOS diagnostics. This likely relates to the fact that $\mathrm{AMH}$, produced by follicular granulosa cells, has emerged as a promising biomarker of ovarian reserve and "reproductive age," correlating with follicle count $(49,50)$. Nevertheless, when analyzing PCOS and $\mathrm{AMH}$ together in a multiple regression model, only AMH remained an independent determinant of circulating prorenin.

Remarkably, preconception BMI associated negatively with prorenin. Because this relation was not observed in 
nonpregnant women (51), it may be pregnancy specific. A logical link would be adipocyte-derived leptin, which upregulates aldosterone (thereby suppressing RAS activation) (52), but differences in diet, oxidative stress, or epigenetics need to be considered as well $(53,54)$. Indeed, the RAAS regulates angiogenesis and vasculogenesis, which are major features of reproduction, known to be affected by epigenetic pathways $(55,56)$. Moreover, angiotensin II induces oxidative stress, and this affects "reproductive aging," a strong determinant of ovarian (endocrine) health (57). From this point of view, it is not surprising that BMI in a multiple regression model no longer remained an independent determinant of prorenin. This model did support a positive association between prorenin and age. This has been noted before (51). An additional explanation of this association in the present population might be that women of advanced reproductive age present earlier with infertility, requiring prorenin-upregulating COS treatment.

We show in the Predict cohort that pregnancies conceived in the absence of a CL were more disposed to develop preeclampsia, which confirms the data of the POFI cohort (14). It is important to note that RAAS activity is known to be suppressed in this condition $(26,35)$. Of interest, the 7 women in the Predict Study who later developed preeclampsia already at 9 weeks of gestation displayed the lowest renin/total renin ratios. This suggests that the RAAS suppression in preeclampsia might originate very early in pregnancy (35).

The strength of this study is its longitudinal periconceptional design, resulting in identical observations in 2 tertiary referral hospitals in the United States and the Netherlands, despite differences in the underlying reproductive disorders, fertility treatment, and patient characteristics. To the best of our knowledge, our study is the largest reporting RAAS determinants concentrations in early pregnancy. It demonstrates that prorenin release depends on CL number and total follicle diameter, and correlates with the circulating progesterone and relaxin levels. It raises the possibility that the type of ovarian stimulation, PCOS, AMH, and BMI are additional determinants of RAAS activation in pregnancy. Nevertheless, it does not allow conclusions on the exact contribution of prorenin. A further limitation is that, given the nature of our participants, the study denotes a high internal and low generalizability (ie, external validity). Residual confounding cannot be excluded entirely. Future studies should now expand the current patient numbers and study RAAS alterations in relation to maternal cardiovascular, renal, osmoregulatory, and volume (mal)adaptation parameters, and indicators of placental development and fetal growth. Ultimately, such understanding might allow us to induce the degree of
RAAS activation that is optimal for the individual pregnant woman, making use of highly personalized IVF protocols.

\section{Acknowledgments}

The authors thank all participants and hospitals that supported the collection of data for the POFI Study and the Predict Study. The authors on behalf of the POFI Study thank Delila Adams for administrative support and Raquel Fleischmann, who participated in recruiting women and entering data. The Rotterdam Periconception Cohort team, in particular Igna Reijnders, Annemarie Mulders, Anton Koning, and Damiat Aoulad Fares, are acknowledged for logistics and data collection.

Financial Support: The prospective POFI cohort study at Stanford was funded by P01 HD 065647-01A1 from the National Institute of Child Health and Human Development (V.L.B., principal investigator, and K.P.C., project director and principal investigator). The use of REDCap for data management was supported by a Stanford CTSA award from the National Institutes of Health/National Center for Research Resources (number UL1 TR001085). The Rotterdam Periconception cohort was funded by the Department of Obstetrics and Gynecology of the Erasmus MC, University Medical Centre, Rotterdam, the Netherlands. R.E.W. received a Van Walree Grant of the Royal Netherlands Academy of Arts and Sciences.

\section{Additional Information}

Correspondence and Reprint Requests: R.P.M. SteegersTheunissen, MD, PhD, Erasmus MC, University Medical Center Rotterdam, Department of Obstetrics and Gynecology, Room EE2271a, PO Box 2040, 3000 CA Rotterdam, the Netherlands. E-mail: r.steegers@erasmusmc.nl.

Disclosure Summary: The authors have nothing to disclose.

Data Availability: All data generated or analyzed during this study are included in this published article or in the data repositories listed in "References."

\section{References}

1. Conrad KP, Baker VL. Corpus luteal contribution to maternal pregnancy physiology and outcomes in assisted reproductive technologies. Am J Physiol Regul Integr Comp Physiol. 2013;304(2):R69-R72.

2. Csapo AI, Pulkkinen MO, Wiest WG. Effects of luteectomy and progesterone replacement therapy in early pregnant patients. Am J Obstet Gynecol. 1973;115(6):759-765.

3. Derkx FH, Alberda AT, de Jong FH, Zeilmaker FH, Makovitz JW, Schalekamp MA. Source of plasma prorenin in early and late pregnancy: observations in a patient with primary ovarian failure. J Clin Endocrinol Metab. 1987;65(2):349-354.

4. McGuane JT, Debrah JE, Debrah DO, et al. Role of relaxin in maternal systemic and renal vascular adaptations during gestation. Ann N Y Acad Sci. 2009;1160:304-312.

5. Conrad KP. Maternal vasodilation in pregnancy: the emerging role of relaxin. Am J Physiol Regul Integr Comp Physiol. 2011;301(2):R267-R275.

6. Sealey JE, Atlas SA, Glorioso N, Manapat H, Laragh JH. Cyclical secretion of prorenin during the menstrual cycle: 
synchronization with luteinizing hormone and progesterone. Proc Natl Acad Sci U S A. 1985;82(24):8705-8709.

7. Sealey J. The ovarian renin-angiotensin system. Front Neuroendocrinol. 1990;11:213-237.

8. Speth RC, Daubert DL, Grove KL. Angiotensin II: a reproductive hormone too? Regul Pept. 1999;79(1):25-40.

9. Irani RA, Xia Y. The functional role of the reninangiotensin system in pregnancy and preeclampsia. Placenta. 2008;29(9):763-771.

10. August P, Sealey, JB. Renin-angiotensin system in normal and hypertensive pregnancy and in ovarian function. In: Laragh JH, Brenner BM, eds. Hypertension: Pathophysiology, Diagnosis, and Management. New York: The Raven Press; 1990:1761-1778.

11. Conrad KP, Graham GM, Chi YY, et al. Potential influence of the corpus luteum on circulating reproductive and volume regulatory hormones, angiogenic and immunoregulatory factors in pregnant women. Am J Physiol Endocrinol Metab. 2019;317(4):E677-E685.

12. Conrad KP, Petersen JW, Chi YY, et al. Maternal cardiovascular dysregulation during early pregnancy after in vitro fertilization cycles in the absence of a corpus luteum. Hypertension. 2019;74(3):705-715.

13. Smith MC, Murdoch AP, Danielson LA, Conrad KP, Davison JM. Relaxin has a role in establishing a renal response in pregnancy. Fertil Steril. 2006;86(1):253-255.

14. von Versen-Höynck F, Schaub AM, Chi YY, et al. Increased preeclampsia risk and reduced aortic compliance with in vitro fertilization cycles in the absence of a corpus luteum. Hypertension. 2019;73(3):640-649.

15. Jackson RA, Gibson KA, Wu YW, Croughan MS. Perinatal outcomes in singletons following in vitro fertilization: a metaanalysis. Obstet Gynecol. 2004;103(3):551-563.

16. Keegan DA, Krey LC, Chang HC, Noyes N. Increased risk of pregnancy-induced hypertension in young recipients of donated oocytes. Fertil Steril. 2007;87(4):776-781.

17. von Versen-Höynck F, Narasimhan P, Selamet Tierney ES, et al. Absent or excessive corpus luteum number is associated with altered maternal vascular health in early pregnancy. Hypertension. 2019;73(3):680-690.

18. Ginström Ernstad E, Wennerholm UB, Khatibi A, Petzold M, Bergh C. Neonatal and maternal outcome after frozen embryo transfer: increased risks in programmed cycles. Am J Obstet Gynecol. 2019;221(2):126.e1-126.e18.

19. Floyd EG, von Versen-Höynck F, Liu J, Chi YY, Fleischmann RR, Baker VL. Collection of pregnancy outcome records following infertility—challenges and possible solutions. J Assist Reprod Genet. 2016;33(8):993-999.

20. Steegers-Theunissen RP, Verheijden-Paulissen JJ, van Uitert EM, et al. Cohort profile: the Rotterdam periconceptional cohort (Predict Study). Int J Epidemiol. 2016;45(2):374-381.

21. von Versen-Höynck F, Strauch NK, Liu J, et al. Effect of mode of conception on maternal serum relaxin, creatinine, and sodium concentrations in an infertile population. Reprod Sci. 2019;26(3):412-419.

22. Hohmann FP, Macklon NS, Fauser BC. A randomized comparison of two ovarian stimulation protocols with gonadotropinreleasing hormone $(\mathrm{GnRH})$ antagonist cotreatment for in vitro fertilization commencing recombinant follicle-stimulating hormone on cycle day 2 or 5 with the standard long GnRH agonist protocol. J Clin Endocrinol Metab. 2003;88(1):166-173.

23. Heijnen EM, Eijkemans MJ, De Klerk C, et al. A mild treatment strategy for in-vitro fertilisation: a randomised non-inferiority trial. Lancet. 2007;369(9563):743-749.

24. Batenburg WW, de Bruin RJ, van Gool JM, et al. Aliskirenbinding increases the half life of renin and prorenin in rat aortic vascular smooth muscle cells. Arterioscler Thromb Vasc Biol. 2008;28(6):1151-1157.

25. Roberts JM, August PA, Bakris G, et al. Hypertension in pregnancy. Report of the American College of Obstetricians and Gynecologists' task force on hypertension in pregnancy. Obstet Gynecol.2013;122(5):1122-1131.

26. Verdonk K, Saleh L, Lankhorst S, et al. Association studies suggest a key role for endothelin-1 in the pathogenesis of preeclampsia and the accompanying renin-angiotensin-aldosterone system suppression. Hypertension. 2015;65(6):1316-1323.

27. Menken J, Trussell J, Larsen U. Age and infertility. Science. 1986;233(4771):1389-1394.

28. Barber TM, McCarthy MI, Wass JA, Franks S. Obesity and polycystic ovary syndrome. Clin Endocrinol (Oxf). 2006;65(2):137-145.

29. Laven JS, Mulders AG, Visser JA, Themmen AP, De Jong FH, Fauser BC. Anti-Müllerian hormone serum concentrations in normoovulatory and anovulatory women of reproductive age. $J$ Clin Endocrinol Metab. 2004;89(1):318-323.

30. Itskovitz J, Sealey JE, Glorioso N, Rosenwaks Z. Plasma prorenin response to human chorionic gonadotropin in ovarian-hyperstimulated women: correlation with the number of ovarian follicles and steroid hormone concentrations. Proc Natl Acad Sci U S A. 1987;84(20):7285-7289.

31. Sealey JE, Glorioso N, Itskovitz J, Troffa C, Cholst I, Rosenwaks Z. Plasma prorenin during early pregnancy: ovarian secretion under gonadotropin control? J Hypertens Suppl. 1986;4(5):S92-S95.

32. Derkx FH, Alberda AT, Zeilmaker GH, Schalekamp MA. High concentrations of immunoreactive renin, prorenin and enzymatically-active renin in human ovarian follicular fluid. $\mathrm{Br}$ J Obstet Gynaecol. 1987;94(1):4-9.

33. Schalekamp MA, Derkx FH, Deinum J, Danser AJ. Newly developed renin and prorenin assays and the clinical evaluation of renin inhibitors. J Hypertens. 2008;26(5):928-937.

34. Derkx FH, Schalekamp MA. Human prorenin: pathophysiology and clinical implications. Clin Exp Hypertens A. 1988;10(6):1213-1225.

35. Verdonk K, Visser W, Van Den Meiracker AH, Danser AH. The renin-angiotensin-aldosterone system in pre-eclampsia: the delicate balance between good and bad. Clin Sci (Lond). 2014;126(8):537-544.

36. Pessôa BS, Slump DE, Ibrahimi K, et al. Angiotensin II type 2 receptor- and acetylcholine-mediated relaxation: essential contribution of female sex hormones and chromosomes. Hypertension. 2015;66(2):396-402.

37. Uysal F, Ozturk S, Akkoyunlu G. Superovulation alters DNA methyltransferase protein expression in mouse oocytes and early embryos. I Assist Reprod Genet. 2018;35(3):503-513. 
38. Do YS, Sherrod A, Lobo RA, et al. Human ovarian theca cells are a source of renin. Proc Natl Acad Sci U S A. 1988;85(6):1957-1961.

39. Richards JS, Ren YA, Candelaria N, Adams JE, Rajkovic A. Ovarian follicular theca cell recruitment, differentiation, and impact on fertility: 2017 update. Endocr Rev. 2018;39(1):1-20.

40. Heijnen EM, Eijkemans MJ, Hughes EG, Laven JS, Macklon NS, Fauser BC. A meta-analysis of outcomes of conventional IVF in women with polycystic ovary syndrome. Hum Reprod Update. 2006;12(1):13-21.

41. Poisner AM, Thrailkill K, Poisner R, Handwerger S. Relaxin stimulates the synthesis and release of prorenin from human decidual cells: evidence for autocrine/paracrine regulation. J Clin Endocrinol Metab. 1990;70(6):1765-1767.

42. Sealey JE, Itskovitz-Eldor J, Rubattu S, et al. Estradiol- and progesterone-related increases in the renin-aldosterone system: studies during ovarian stimulation and early pregnancy. J Clin Endocrinol Metab. 1994;79(1):258-264.

43. Schunkert H, Danser AH, Hense HW, Derkx FH, Kürzinger S, Riegger GA. Effects of estrogen replacement therapy on the renin-angiotensin system in postmenopausal women. Circulation. 1997;95(1):39-45.

44. Batenburg WW, Lu X, Leijten F, Maschke U, Müller DN, Danser AH. Renin- and prorenin-induced effects in rat vascular smooth muscle cells overexpressing the human (pro)renin receptor: does (pro)renin-(pro)renin receptor interaction actually occur? Hypertension. 2011;58(6):1111-1119.

45. Jaatinen TA, Matinlauri I, Anttila L, Koskinen P, Erkkola R, Irjala K. Serum total renin is elevated in women with polycystic ovarian syndrome. Fertil Steril. 1995;63(5):1000-1004.

46. Sealey JE, Cholst I, Glorioso N, et al. Sequential changes in plasma luteinizing hormone and plasma prorenin during the menstrual cycle. J Clin Endocrinol Metab. 1987;65(1):1-5.

47. Magoffin DA. Ovarian theca cell. Int J Biochem Cell Biol. 2005;37(7):1344-1349.
48. Nelson VL, Legro RS, Strauss JF III, McAllister JM. Augmented androgen production is a stable steroidogenic phenotype of propagated theca cells from polycystic ovaries. Mol Endocrinol. 1999;13(6):946-957.

49. de Vet A, Laven JS, de Jong FH, Themmen AP, Fauser BC. Antimüllerian hormone serum levels: a putative marker for ovarian aging. Fertil Steril. 2002;77(2):357-362.

50. Kevenaar ME, Themmen AP, Rivadeneira F, et al. A polymorphism in the AMH type II receptor gene is associated with age at menopause in interaction with parity. Hum Reprod. 2007;22(9):2382-2388

51. Danser AH, Derkx FH, Schalekamp MA, Hense HW, Riegger GA, Schunkert H. Determinants of interindividual variation of renin and prorenin concentrations: evidence for a sexual dimorphism of (pro)renin levels in humans. J Hypertens. 1998;16(6):853-862.

52. Huby AC, Antonova G, Groenendyk J, et al. Adipocyte-derived hormone leptin is a direct regulator of aldosterone secretion, which promotes endothelial dysfunction and cardiac fibrosis. Circulation. 2015;132(22):2134-2145.

53. Mistry HD, Kurlak LO, Broughton Pipkin F. The placental renin-angiotensin system and oxidative stress in pre-eclampsia. Placenta. 2013;34(2):182-186.

54. Reijnders IF, Mulders AGMGJ, van der Windt M, Steegers EAP, Steegers-Theunissen RPM. The impact of periconceptional maternal lifestyle on clinical features and biomarkers of placental development and function: a systematic review. Hum Reprod Update. 2019;25(1):72-94.

55. Smith SK. Angiogenesis and reproduction. BJOG. 2001;108(8):777-783.

56. Steegers-Theunissen RP, Steegers EA. Nutrient-gene interactions in early pregnancy: a vascular hypothesis. Eur J Obstet Gynecol Reprod Biol. 2003;106(2):115-117.

57. Lu J, Wang Z, Cao J, Chen Y, Dong Y. A novel and compact review on the role of oxidative stress in female reproduction. Reprod Biol Endocrinol. 2018;16(1):80. 